\title{
Clinical factors affecting the determination of radiotherapy- induced skin toxicity in breast cancer
}

\author{
Elisa Eugenia Córdoba ${ }^{1,2}$, Ezequiel Lacunza ${ }^{3}$, Alba Mabel Güerci ${ }^{1,2}$ \\ ${ }^{1}$ Department of Physics, School of Exact Sciences, National University of La Plata, Argentina. \\ ${ }^{2}$ Veterinary Genetics Institute (National Scientific and Technical Research Council-National University of La Plata) School of Veterinary Sciences, \\ La Plata, Argentina. \\ ${ }^{3}$ Basic and Applied Immunological Research Center, School of Medicine, National University of La Plata, Argentina.
}

Received: June 25, 2020

Revised: October 9, 2021

Accepted: October 19, 2021

\section{Correspondence:}

Elisa Eugenia Córdoba

Veterinary Genetics Institute (National

Scientific and Technical Research

Council-National University of La

Plata) School of Veterinary Sciences,

La Plata, Calle 60 y 118 s/n (1900) La

Plata, Buenos Aires, Argentina.

Tel: +54-2215629081

E-mail: elisaecordoba@gmail.com ORCID:

https://orcid.org/0000-0001-7221-2625
Purpose: Radiotherapy is essential for the treatment of breast cancer (BC). However, adverse effects may occur in healthy tissue, during treatment and even after several months. Although it is known that this clinical radiosensitivity is multifactorial, the factors involved are unknown yet. In this study, we evaluated the effect of these factors on the development of radiodermatitis in patients undergoing radiotherapy.

Materials and Methods: Demographic and lifestyle data collected during face-to-face interviews of $122 \mathrm{BC}$ patients and data from clinical records were investigated. Most patients underwent conventional three-dimensional radiotherapy treatment. A total dose of $50 \mathrm{~Gy}$ was administered (2 Gy/day), followed by a boost in a tumor bed with a total dose of $18 \mathrm{~Gy}$ ( $2 \mathrm{~Gy} /$ day). Radiotoxicity was evaluated weekly using the Radiation Therapy Oncology Group classification system (range, 0 to 4, according to the severity).

Results: In the present study, 75.4\% of patients presented acute skin toxic effects with different degrees of severity. In 25\% of cases, these effects manifested at the end of the fourth week at a cumulative dose of $40 \mathrm{~Gy}$. The association of grade $\geq 2$ acute skin reactions with body mass index (BMI) and breast size and between grade 3-4 and age was positive compared with controls. However, the role of the other factors could not be confirmed.

Conclusion: Analysis of the factors related to individual radiosensitivity suggests that age, BMI and breast size play an important role in the development of acute skin toxicity during treatment. Particular attention to patients who present these characteristics would help to control treatment effectiveness and therefore optimize their quality of life.

Keywords: Radiotherapy, Breast neoplasms, Radiation injuries, Radiodermatitis

\section{Introduction}

Although the quality of life of the patient is an essential component when considering cancer therapies, there are not many studies on the adverse effects of radiotherapy (RT) $[1,2]$. While ionizing radiation is an important tool for the treatment of several tumors, it usually induces pathological effects on healthy tissue, which limit the therapeutic dose. Since tissue radiosensitivity depends on cell proliferation, tissues with a greater regenerative capacity, such as the skin, will be more affected by irradiation [3]. In this way, it is understood that radiodermatitis is the most significant side effect of RT for breast cancer (BC) and responsible for the physical and emotional discomfort of the patient that interferes with the treatment $[1,2,4]$.

These side effects of RT respond to a dynamic process that may include lesions of increasing complexity $[5,6]$ and are classified ac- 
cording to their time of onset. Acute or early effects occur during the course of treatment or up to 6 months later and are generally reversible [7]. They manifest as erythema, dermatitis, wet or dry scaling, depilation, and ulceration [7-9]. On the other hand, late effects occur from 6 months to years after the end of the treatment, due to irreparable damage to the dermis. They include atrophy, fibrosis, telangiectasia, and pigmentation abnormalities $[3,10,11]$.

Recent studies have emphasized the role of the RT-induced inflammatory response in the pathogenesis of normal tissue. Cellular injury resulting from oxidative stress produces infiltration of lymphocytes and macrophages with the release of various pro-inflammatory cytokines and fibroblast stimulation. This, in turn, leads to the production of reactive oxygen species (ROS) and generates a cyclical response that is perpetuated over time $[12,13]$. This inflammation process could be responsible for dermal edema and skin erythema due to permeability and capillary dilation. Furthermore, dryness and hair removal are the result of the destruction of sebaceous glands and hair follicles in the dermal layer. Hyperpigmented skin occurs by stimulation of epidermal melanocytes. Dry desquamation develops when stem cells from the basal layer are depleted in the treated area, while wet desquamation occurs when all stem cells are eradicated from the basal layer and are distinguished by exposure of the dermis and serous exudation. Some patients may also experience fatigue [14-16].

Despite the use of uniform protocols, this patient response ranges from mild to severe toxicity based on individual radiosensitivity $[8,17,18]$. Although genetic factors are elementary in their establishment and the main signaling pathways have been identified by radiogenomics, numerous elements must be considered when addressing this multifactorial trait. These are mainly related to physics (radiation dose, dose rate, inhomogeneity of dose and treatment volume), treatment (interaction with other therapies such as surgery, chemotherapy and hormonal therapy) and the patient (age, hemoglobin levels, smoking, comorbidities such as diabetes, obesity and vascular diseases) $[10,18,19]$.

Understanding that beyond the technological advances that made it possible to improve the administration of radiation, the development of radiotoxicity in normal tissue is always possible, understanding the individual differences in this response, can allow the adaptation of medical care to the needs of each patient and thus achieve a better therapeutic result $[2,20,21]$. Bearing in mind the prevalence of $B C$ in our region, the objective of this study was to expand our knowledge of the factors involved in radiotoxicity, given the profound impact that this trait can have for some patients.

\section{Materials and Methods}

\section{Study sample}

Patients with $B C$ from the city of La Plata and its surroundings undergoing RT at the Instituto de Terapia Radiante, Centro Integrado de Oncología (CIO), La Plata, Argentina, were studied. The sample included 122 individuals receiving conservative treatment/ post-mastectomy therapy. None of the patients had previously received RT.

Face-to-face interviews were used for patient data collection on personal, demographic and lifestyle data: age, place of birth, ethnicity, smoking habit, alcohol consumption, weight, height, size of the breast, comorbidities such as diabetes, hypertension and anemia, and family history of BC or any other type of neoplasia. Other relevant information was collected from patient clinical records.

\section{Ethical considerations}

The study was conducted exclusively with patients who gave their consent. Each patient was informed about the protocol of the study, both verbally and in writing in accordance with the Declaration of Helsinki, promulgated by the World Medical Association. The main project of the present study was approved by the Ad-hoc Committee of Ethics of the Human Genetics Association of the city of Mar del Plata, Buenos Aires, Argentina.

\section{Radiation therapy}

Most BC patients (98/122) underwent three-dimensional (3D) conservative treatment, after quadrantectomy. Whole-breast RT was delivered with a linear accelerator of 4 or 6 MV photons (Clinac; Varian Medical Systems, Palo Alto, CA, USA) in conventional 50 Gy doses fractionated in daily 2 Gy doses. The treatment was followed by a 12-18 Gy boost to the tumor bed in fractionated doses of 2 Gy/day in $85.7 \%$ of cases (84/98). Besides hypofractionated RT treatment was administered to 9/122 BC patients (after quadrantectomy) over age 60 years and at an early tumor stage in $42.56 \mathrm{~Gy}$ doses, fractionated at $2.66 \mathrm{~Gy} /$ day and without boost. Standard RT was delivered to the costal wall of 12/122 post-mastectomy patients using tangential fields as daily 1.8-2 Gy doses, up to a total dose of $50 \mathrm{~Gy}$. In most cases, the axillary-supraclavicular cavity was also treated. Finally, two patients without surgical intervention were the exception: one with a conventional treatment palliative $60 \mathrm{~Gy}$ plus $50.4 \mathrm{~Gy}$ in the axillo-supraclavicular cavity and another as a pre-surgical treatment in $50 \mathrm{~Gy}$ doses.

\section{Radiotoxicity evaluation}

Acute radiotoxicity was analyzed by the team of radio-oncologists at CIO-La Plata, who weekly recorded the clinical evolution of 
treatment. To control the appearance of radio-induced side effects, moisturizing cream with vitamin A was prescribed. The classification system of the Radiation Therapy Oncology Group (RTOG) was used to evaluate the severity of acute skin reactions [22] (Table 1). The development of grade $\geq 2$ acute side effects was indicative of patients with greater sensitivity, while individuals with minimal or no skin reaction (Grades 0 and 1 ) were included in the control group. The occurrence and severity of acute skin reactions were determined during and after the RT treatment, taking into account the highest degree of radiotoxicity for the statistical analysis. In case of pruritus or eczema, corticosteroids or allantoin were prescribed and sodium borate diluted 6:1000 was indicated to treat moist dermatitis.

\section{Statistical analysis}

The association of each variable with radiodermatitis was analyzed using the $\chi^{2}$ test. Relative risk was estimated by calculating the odds ratio (OR) with 95\% and 99\% confidence intervals. Multivariate analysis was carried out using principal component analysis (PCA) [23]. The SPSS program version 22 (IBM SPSS, Armonk, NY, USA) was used for data analysis.

\section{Results}

The demographic and clinical characteristics of the study sample are shown in Table 2. Mean BC patient age was $59.6 \pm 12.5$ years (range, 26 to 85 years; mode, 52 and 62 years; median, 61.5 years).

Analysis of BC family history showed that $38 \%$ of patients had first- and second-degree relatives with $\mathrm{BC}$, while $30 \%$ reported no history of any type of oncological pathology. Ancestry data analysis revealed that $60 \%$ of patients reported a European origin, mostly Italian (33\%) and Spanish (18\%), followed by Amerindians (39\%) and other ethnic groups (1\%).

Information about the clinical manifestations of radiotoxicity recorded by radiological oncologists demonstrated that $75.4 \%$ of patients (92/122) presented acute cutaneous toxic effects with dif- ferent degrees of severity, while $24.6 \%$ of women (30/122) remained without skin changes (Fig. 1). On the other hand, these side effects could be seen in $8.69 \%$ of patients (8/92) after the end of the first week of treatment together with erythema and pruritus, at a cumulative dose of $10 \mathrm{~Gy}$. In 25\% of cases (23/92), these effects manifested at the end of the fourth week of treatment at a dose of $40 \mathrm{~Gy}$, showing a decreasing trend $(9.78 \%, 9 / 92)$ towards the seventh week at $70 \mathrm{~Gy}$. These results showed the normal distribution of this quantitative trait (Fig. 2).

The association analysis of the most relevant clinical variables and radiosensitivity showed that the age range of $46.8 \%$ of patients with grade $\geq 2$ acute radiodermatitis was $56-70$ years. When we analyze the patients by degree of radiodermatitis and age, young patients ( $<59.6$ years) and older patients ( $\geq 59.6$ years), it was observed that there is a tendency to develop more severe effects with increasing to age (Fig. 3). However, it was not significant.

The association of grade $\geq 2$ acute skin reactions with body mass index $(\mathrm{BMI})$ and breast size was positive $(\mathrm{OR}=2.87, \mathrm{p}=0.026$ and $\mathrm{OR}=4.62, \mathrm{p}=0.005$, respectively). Patients with medium and large breasts showed 4.62 times higher risk of developing radiodermatitis than those with small breast. Likewise, 69\% of patients who developed grade $\geq 2$ radiodermatitis were overweight or obese $(\mathrm{BMI}>25)$. Obese patients presented 2.87 times more risk of man-

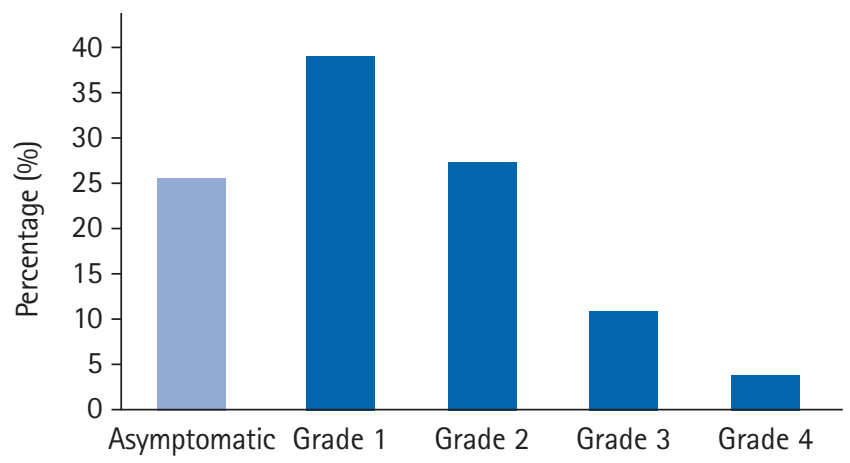

Fig. 1. Percentage of breast cancer patients with radiodermatitis developed during treatment.

Table 1. Severity of acute skin reactions according to the EORTC/RTOG criteria

\begin{tabular}{|c|c|c|c|c|}
\hline \multicolumn{5}{|c|}{ Acute radiodermatitis } \\
\hline Grade 0 & Grade 1 & Grade 2 & Grade 3 & Grade 4 \\
\hline \multirow[t]{5}{*}{ No changes are observed } & Follicular, mild diffuse erythema & Marked erythema & Confluent moist desquamation & Haemorrhages \\
\hline & Epilation & Moist desquamation in & Severe edema & Ulceration \\
\hline & Pruritus & circumscribed foci & & Necrosis \\
\hline & Dry desquamation & Moderate edema & & \\
\hline & Decreased sweating & & & \\
\hline
\end{tabular}

RTOG, Radiation Therapy Group; EORTC, European Organization for Research and Treatment of Cancer.

Adapted from Cox et al. [22]. 
Table 2. Clinical characteristics of breast cancer patients

\begin{tabular}{|c|c|}
\hline Characteristic & Value \\
\hline Age (yr) & $59.6 \pm 12.5(26-85)$ \\
\hline \multicolumn{2}{|l|}{ Sex } \\
\hline Female & $122(100)$ \\
\hline \multicolumn{2}{|l|}{ Tumour location } \\
\hline Left breast & $74(60.7)$ \\
\hline Right breast & $48(39.3)$ \\
\hline \multicolumn{2}{|l|}{ Histological type of tumor } \\
\hline DCIS & $12(10)$ \\
\hline LCIS & $4(3)$ \\
\hline IDC & $85(71)$ \\
\hline ILC & $10(8)$ \\
\hline IDLC & $6(5)$ \\
\hline Metaplastic & $2(2)$ \\
\hline ITC & $1(1)$ \\
\hline \multicolumn{2}{|l|}{ Neo-adjuvant chemotherapy } \\
\hline Yes & $35(31)$ \\
\hline No & $78(69)$ \\
\hline \multicolumn{2}{|l|}{ Tamoxifen } \\
\hline Yes & $68(69)$ \\
\hline No & $30(31)$ \\
\hline \multicolumn{2}{|l|}{ Diabetes } \\
\hline Yes & $14(12)$ \\
\hline No & $103(88)$ \\
\hline \multicolumn{2}{|l|}{ Hypertension } \\
\hline Yes & $42(36)$ \\
\hline No & $75(64)$ \\
\hline \multicolumn{2}{|l|}{ Smoking } \\
\hline Yes & $32(28)$ \\
\hline No & $81(72)$ \\
\hline \multicolumn{2}{|l|}{ Alcohol consumption ${ }^{\text {a) }}$} \\
\hline Yes & $10(9)$ \\
\hline No & $103(91)$ \\
\hline \multicolumn{2}{|l|}{ BMI $\left(\mathrm{kg} / \mathrm{m}^{2}\right)$} \\
\hline$<25$ (normal) & $46(41)$ \\
\hline$\geq 25$ (overweight and obesity) & $67(59)$ \\
\hline \multicolumn{2}{|l|}{ Breast size $\mathrm{e}^{\mathrm{b})}$} \\
\hline Small & $26(24)$ \\
\hline Medium & $49(46)$ \\
\hline Large & $32(30)$ \\
\hline
\end{tabular}

Values are presented as median (range) or number (\%).

BMI, body mass index; DCIS, ductal carcinoma in situ; LCIS, lobular carcinoma in situ; IDC, invasive ductal carcinoma; ILC, invasive lobular carcinoma; IDLC, invasive ductal-lobular carcinoma; ITC, invasive tubular carcinoma.

a) Alcohol consumption refers to consumption during meals.

${ }^{b}$ The size of the breast was measured by direct visual evaluation.

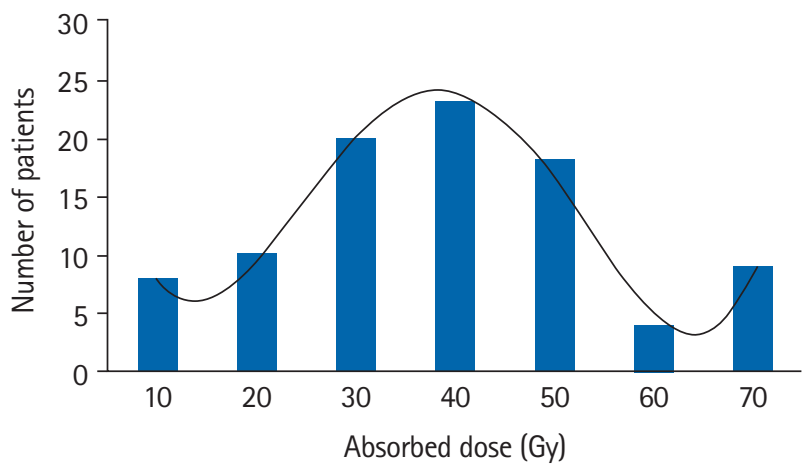

Fig. 2. Radiodermatitis associated to the dose absorbed. A large number of patients developed radiodermatitis to the approximately 40 Gy dose during the fourth week of treatment.

ifesting radiodermatitis than patients with a normal BMI (Table 3).

Regarding the association of smoking habit, alcohol consumption, multivitamin intake, anemia, hypertension, diabetes, skin color, chemotherapy, tamoxifen and type of planning with development of acute radiodermatitis, no significant differences were observed between both groups of patients ( $<<0.05$ ). The PCA performed with some of these variables showed that BMI and breast size contributed to the development of acute skin radiotoxicity during RT treatment, while the contribution of the other factors could not be confirmed (Fig. 4).

\section{Discussion and Conclusion}

Individual radiosensitivity is a complex trait with multifactorial and polygenic inheritance. Therefore, the influence of the environment on its phenotypic expression is fundamental. Radiosensitivity refers to the level of toxicity in healthy tissue of individuals subjected to $\mathrm{RT}$, since the development of adverse effects in patients undergoing similar protocols is variable $[8,18]$. This could be attributed to cell depletion and inhibition of cell repopulation in rapidly renewing tissues, such as the skin [24,25].

Although significant efforts have been made to evaluate radiodermatitis in $\mathrm{BC}$ patients, evidence in the literature is scarce compared with that on other symptoms related to side effects and quality of life [26], probably because most RT medical centers do not usually carry out registry of toxicity as a routine [18]. Therefore, our attention should be focused more on obtaining detailed, accurate and complete information about study participants during a more appropriate follow-up period in order to maximize the chances of finding an association between RT-induced toxicity and its determining factors [10]. Understanding the impact of radiodermatitis on patient quality of life is essential not only for the correct management of cutaneous reactions but also for the development 


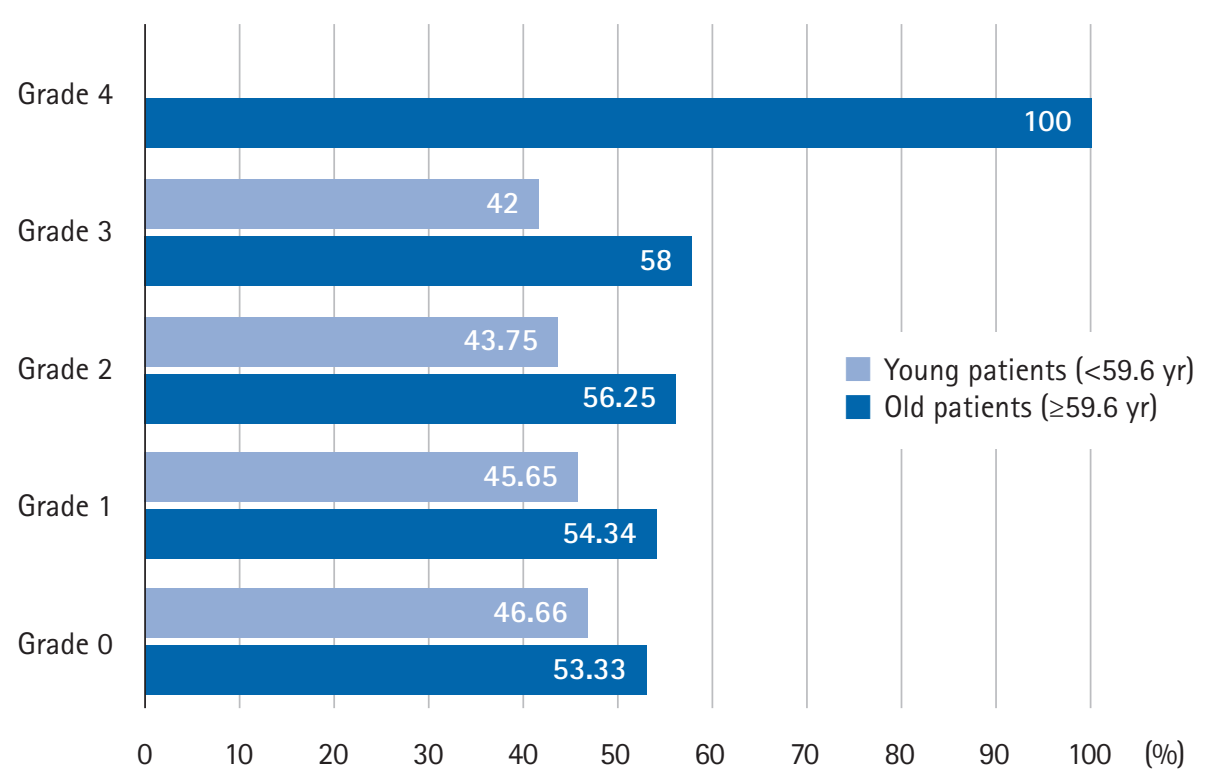

Fig. 3. Percentage of old and young patients (above and below the mean age) according to the different degrees of radiodermatitis.

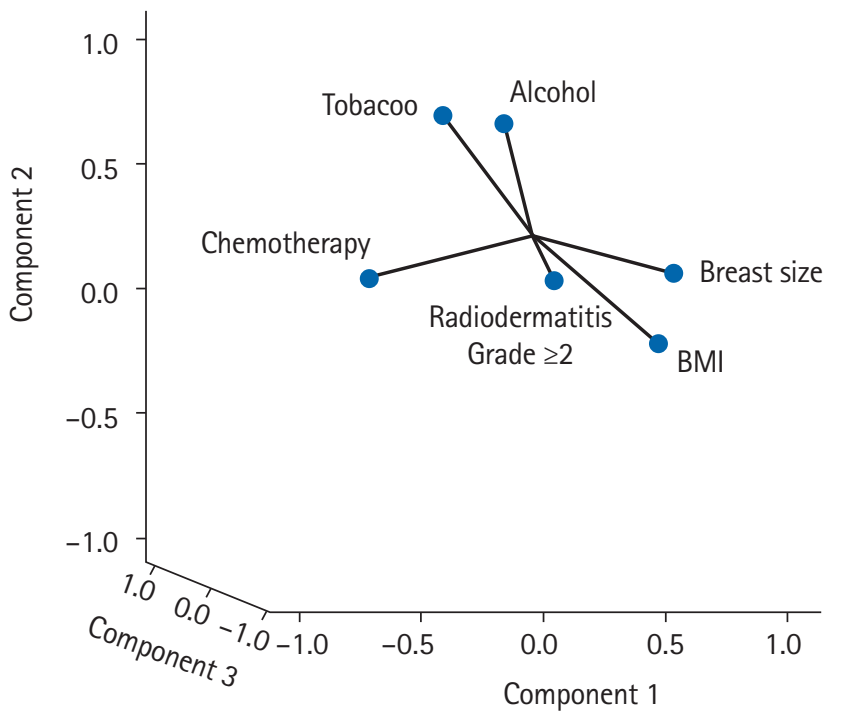

Fig. 4. Principal component analysis. There was a close relationship between patient breast size and body mass index (BMI) with radiodermatitis grade $\geq 2$ developed during the radiotherapy treatment.

of trials that can predict them.

Our results are in agreement with those of Khanna et al. [27], who showed that radiodermatitis was the most commonly observed side effect during RT. Three quarters of the population analyzed presented skin toxicity, with a higher incidence of grades 1 and 2 (85\%) compared with the more severe grades 3 and 4 . Similarly, Schnur et al. [26] argued that most BC patients (74\%) could experience radiodermatitis during the course of RT at high doses. Meanwhile, De Langhe et al. [28] estimated that 10\% of cases suf- fered more severe effects such as moist desquamation or skin ulceration, and Pignol et al. [29] reported higher percentages of severe dermatitis (20\%-25\%).

The results obtained by our group showed that pruritus and erythema events occurred towards the end of the fourth week with 40 Gy doses in 25\% of cases. However, these skin injuries progressed towards the first week in $8.69 \%$ of highly sensitive individuals with cumulative doses that reached $10 \mathrm{~Gy}$ (left side of the curve in Fig. 2). In the most tolerant individuals (9.78\%), these effects were detected at doses greater than $70 \mathrm{~Gy}$ (right side of the curve in Fig. 2). Thus, individual radiosensitivity had a normal distribution and coincided with the theoretical description presented by Burnet et al. [30] and Barnett et al. [10].

In terms of development of RT-induced injuries, age is usually considered a risk factor [7]. However, there is contradictory evidence about its influence on the prevalence and severity of this feature [31-33]. In the present study, median age was not significantly different in patients with grade $\geq 2$ radiotoxicity and asymptomatic patients with grade 1 radiotoxicity, arguing in favor of reports suggesting that RT is well tolerated in elderly patients $[31,32]$. However, when the analysis was performed in asymptomatic patients and grades 3 and 4 radiotoxicity, women older than 59 years had a higher tendency to present severe radiotoxicity (OR $=6.6 ; p=0.02)$. Generally, advanced age is associated with a greater risk of toxicity due to the lower tolerance of healthy tissue as a result of the reduction in blood flow and DNA repair. In this regard, Lilla et al. [34] reported that the progressive reduction of functional reserves due to the depletion of tissue stem cells could 
Table 3. Association of potential risk factors with skin radiotoxicity

\begin{tabular}{|c|c|c|c|c|c|c|}
\hline & \multirow{2}{*}{$n$} & \multicolumn{2}{|c|}{ Radiodermatitis } & \multirow{2}{*}{$\mathrm{OR}$} & \multirow{2}{*}{$\mathrm{Cl}$} & \multirow{2}{*}{$p$-value } \\
\hline & & Grade 0-1 & Grade $\geq 2$ & & & \\
\hline \multicolumn{7}{|l|}{ Diabetes } \\
\hline No & 103 & 64 & 39 & 1 & & \\
\hline Yes & 14 & 9 & 5 & 0.91 & $0.28-2.91$ & 0.87 \\
\hline \multicolumn{7}{|l|}{ Hypertension } \\
\hline No & 75 & 50 & 25 & 1 & & \\
\hline Yes & 42 & 23 & 19 & 1.2 & $0.76-3.58$ & 0.20 \\
\hline \multicolumn{7}{|l|}{ Breast size } \\
\hline Small & 26 & 22 & 4 & 1 & & \\
\hline Medium & 49 & 28 & 21 & 4.1 & $1.23-13.48$ & $0.016^{*}$ \\
\hline Large & 32 & 16 & 16 & 5.5 & $1.54-19.60$ & $0.005^{*}$ \\
\hline Medium-large & 81 & 44 & 37 & 4.62 & $1.46-14.63$ & $0.005^{*}$ \\
\hline \multicolumn{7}{|l|}{$\mathrm{BMI}$} \\
\hline Normal & 46 & 33 & 13 & 1 & & \\
\hline Overweight & 35 & 22 & 13 & 1.5 & $0.58-3.83$ & 0.39 \\
\hline Obesity & 32 & 15 & 17 & 2.87 & $1.11-7.40$ & $0.026^{*}$ \\
\hline \multicolumn{7}{|l|}{ Skin color } \\
\hline Brown & 74 & 4 & 4 & 1 & & \\
\hline Medium & 31 & 21 & 10 & 0.68 & $1.69-2.88$ & 0.42 \\
\hline Light & 8 & 44 & 30 & 0.68 & $0.15-2.94$ & 0.60 \\
\hline Medium-light & 39 & 65 & 40 & 0.61 & $0.14-2.59$ & 0.50 \\
\hline \multicolumn{7}{|l|}{ Chemotherapy } \\
\hline No & 80 & 53 & 27 & 1 & & \\
\hline Yes & 35 & 19 & 16 & 1.65 & $0.73-3.71$ & 0.22 \\
\hline \multicolumn{7}{|l|}{ Type of treatment } \\
\hline $2 D$ & 9 & 6 & 3 & 1 & & \\
\hline $3 \mathrm{D}$ & 113 & 69 & 44 & 1.27 & $0.30-5.36$ & 0.73 \\
\hline \multicolumn{7}{|l|}{ Alcohol } \\
\hline No & 103 & 65 & 38 & 1 & & \\
\hline Yes & 10 & 5 & 5 & 1.71 & $0.46-6.29$ & 0.41 \\
\hline \multicolumn{7}{|l|}{ Smoking } \\
\hline No & 81 & 50 & 31 & 1 & & \\
\hline Yes & 32 & 20 & 12 & 0.96 & $0.41-2.25$ & 0.93 \\
\hline
\end{tabular}

$\mathrm{BMI}$, body mass index; 2D, two-dimensional; 3D, three-dimensional; $\mathrm{OR}$, odds ratio; $\mathrm{Cl}$, confidence interval. *p $<0.05$.

increase tissue damage and the risk of complications.

Breast size was one of the first characteristics known to influence acute skin toxicity. Several studies report that it interferes with the incidence and severity of radiation of these effects [35]. Larger breasts require the application of higher radiation doses to reach the desired dose in deeper structures $[32,36]$. In addition, it has also been shown that BMI is associated with the development of individual radiosensitivity [9]. In this study, breast size and BMI were associated with an increased risk of developing radiodermatitis $(O R=4.62, p=0.005$ and $O R=2.87, p=0.026$, respectively). In agreement with other studies [35,37], our results corroborate that patients with medium and large breasts showed more severe skin reactions. With respect to BMI, Twardella et al. [38] reported that overweight/obesity (BMI $\geq 25$ ) increased the likelihood of acute radiotoxicity. This was also seen in the current study, since obese women had 2.87 times increased risk compared with normonourished individuals, probably attributable to a lower healing efficiency due to poor vascularization of adipose tissue that compromises healing [39]. Obesity can also cause excessive wear of the skin through increased friction in movement, which would cause abrasion.

The association between smoking and radiodermatitis was not significant, probably due to the low incidence of smokers in this study $(28 \%, 32 / 81)$. However, there is evidence that smoking in- 
creases clinical radiosensitivity in breast as well as in pelvic and head and neck cancer $[35,36]$. Furthermore, smoking may exacerbate radiodermatitis by impairing wound healing [40].

A study of 348 BC patients who underwent RT showed a decrease in acute skin toxicity in individuals who reported regular consumption of wine before starting treatment [41]. Our results could not corroborate such information because only $9 \%$ of the surveyed patients (10/103) drank alcohol during meals.

Among systemic diseases, diabetes mellitus and hypertension could be associated with a low tolerance to RT [18]. In the present study, despite 36\% of women (42/117) were hypertensive, the severity of skin reactions was not significant. With regard to diabetes, only $12 \%$ of patients $(14 / 117)$ had this condition. As reported in other studies, diabetes has not been shown to be a predisposing factor for adverse skin reactions [7,32]. In agreement with this report, Terrazzino et al. [36] indicated that there is no firm evidence that diabetes mellitus and hypertension represent predisposing factors for acute radiosensitivity [7,31].

In relation to elements concerned with RT treatment, chemotherapeutic agents are considered to enhance the effect of radiation and may increase the risk of secondary effects [42]. Although the use of RT in combination with chemotherapy improves overall survival, an increase in both acute and late toxicity is often observed. Many randomized controlled trials of chemoradiotherapy versus RT alone have shown increased acute, but not late, toxicity with the addition of chemotherapy [43]. Some drugs such as doxorubicin, actinomycin D, cisplatin, fluorouracil, apart from their own characteristic spectrum of side effects, may sensitize normal tissues to radiation damage. Such radiosensitization can occur even when chemotherapy is administered before or after the course of fractionated RT. Previous studies have shown a worse cosmetic result and higher late toxicity when chemotherapy is administered, and this seems to be more pronounced when administered simultaneously with RT [44]. In this study, despite some patients (31\%, 35/113) performed neoadjuvant chemotherapy in addition to the radiant treatment, they did not present increased radiosensitivity compared with those only on RT.

Finally, the findings of this study should be considered in light of some limitations, such as, the small number of patients, and partial data obtained from other variables-estrogen receptor (ER), progesterone receptor (PR), and human epidermal growth factor receptor-2 (HER2/neu), the use of multivitamins, or certain chemotherapy drugs such as Herceptin-that were not fully available and therefore were not included in this study.

In conclusion, although it would be interesting to be able to strengthen the sampling, not only to validate the associations found but also to corroborate some suggestions made, this particu- lar analysis of factors related to individual radiosensitivity suggests that age, BMI, and breast size play an important role in the development of acute skin toxicity during RT treatment. In consequence, the particular attention paid to these patients would help to better control treatment effectiveness and therefore optimize quality of life in oncology patients.

\section{Conflict of Interest}

No potential conflict of interest relevant to this article was reported.

\section{Acknowledgements}

The authors thank to Terapia Radiante Red CIO-La Plata for allowing data and samples collection. We also thank to A. DiMaggio for manuscript correction and editing.

\section{References}

1. Fuzissaki MA, Paiva CE, Oliveira MA, Lajolo Canto PP, Paiva Maia YC. The impact of radiodermatitis on breast cancer patients' quality of life during radiotherapy: a prospective cohort study. J Pain Symptom Manage 2019;58:92-9.

2. Beamer LC, Grant M. Skin-related quality of life among midwestern US community-based women with breast cancer experiencing radiodermatitis. Asia Pac J Oncol Nurs 2019;6:50-6.

3. Fallah $M$, Viklund $E_{1}$ Backman $A$, et al. Plasminogen is a master regulator and a potential drug candidate for the healing of radiation wounds. Cell Death Dis 2020;11:201.

4. Singh M, Alavi A, Wong R, Akita S. Radiodermatitis: a review of our current understanding. Am J Clin Dermatol 2016;17:277-92.

5. Fisher J, Scott C, Stevens R, et al. Randomized phase III study comparing Best Supportive Care to Biafine as a prophylactic agent for radiation-induced skin toxicity for women undergoing breast irradiation: Radiation Therapy Oncology Group (RTOG) 9713. Int J Radiat Oncol Biol Phys 2000;48:1307-10.

6. McQuestion M. Evidence-based skin care management in radiation therapy. Semin Oncol Nurs 2006;22:163-73.

7. Porock $D$, Kristjanson L. Skin reactions during radiotherapy for breast cancer: the use and impact of topical agents and dressings. Eur J Cancer Care (Engl) 1999;8:143-53.

8. West CM, Barnett GC. Genetics and genomics of radiotherapy toxicity: towards prediction. Genome Med 2011;3:52.

9. Kole AJ, Kole L, Moran MS. Acute radiation dermatitis in breast cancer patients: challenges and solutions. Breast Cancer (Dove Med Press) 2017;9:313-23. 
10. Barnett GC, West CM, Dunning AM, et al. Normal tissue reactions to radiotherapy: towards tailoring treatment dose by genotype. Nat Rev Cancer 2009;9:134-42.

11. Lee J, Park W, Choi DH, et al. Patient-reported symptoms of radiation dermatitis during breast cancer radiotherapy: a pilot study. Qual Life Res 2017;26:1713-9.

12.Kim JH, Jenrow KA, Brown SL. Mechanisms of radiation-induced normal tissue toxicity and implications for future clinical trials. Radiat Oncol J 2014;32:103-15.

13. Najafi M, Motevaseli $E_{1}$ Shirazi $A$, et al. Mechanisms of inflammatory responses to radiation and normal tissues toxicity: clinical implications. Int J Radiat Biol 2018;94:335-56.

14. Archambeau J0, Pezner R, Wasserman T. Pathophysiology of irradiated skin and breast. Int J Radiat Oncol Biol Phys 1995;31: 1171-85.

15. Hopewell JW. The skin: its structure and response to ionizing radiation. Int J Radiat Biol 1990;57:751-73.

16. White J, Joiner MC. Toxicity from radiation in breast cancer. In: Small W, Woloschak GE, editors. Radiation toxicity: a practical guide. Boston, MA: Springer; 2008, p. 65-109.

17. Bentzen SM. Preventing or reducing late side effects of radiation therapy: radiobiology meets molecular pathology. Nat Rev Cancer 2006;6:702-13.

18. Health Protection Agency. Human radiosensitivity: report of the independent advisory group on ionising radiation. Chilton, UK: Health Protection Agency; 2013.

19. Kerns SL, Ostrer H, Rosenstein BS. Radiogenomics: using genetics to identify cancer patients at risk for development of adverse effects following radiotherapy. Cancer Discov 2014;4:155-65.

20. Burnet NG, Barnett GC, Elliott RM, et al. RAPPER: the radiogenomics of radiation toxicity. Clin Oncol (R Coll Radiol) 2013; 25:431-4.

21. Schnur JB, Ouellette SC, Dilorenzo TA, Green S, Montgomery GH. A qualitative analysis of acute skin toxicity among breast cancer radiotherapy patients. Psychooncology 2011;20:260-8.

22. Cox JD, Stetz J, Pajak TF. Toxicity criteria of the Radiation Therapy Oncology Group (RTOG) and the European Organization for Research and Treatment of Cancer (EORTC). Int J Radiat Oncol Biol Phys 1995;31:1341-6.

23. Broschat TK. Principal component analysis in horticultural research. HortScience 1979;14:114-7.

24. Stone HB, Coleman CN, Anscher MS, McBride WH. Effects of radiation on normal tissue: consequences and mechanisms. Lancet Oncol 2003;4:529-36.

25. Brush J, Lipnick SL, Phillips T, Sitko J, McDonald JT, McBride WH. Molecular mechanisms of late normal tissue injury. Semin Radiat Oncol 2007;17:121-30.
26. Schnur JB, Love B, Scheckner BL, Green S, Wernicke AG, Montgomery $\mathrm{GH}$. A systematic review of patient-rated measures of radiodermatitis in breast cancer radiotherapy. Am J Clin Oncol 2011;34:529-36.

27. Khanna NR, Kumar DP, Laskar SG, Laskar S. Radiation dermatitis: an overview. Indian J Burns 2013;21:24-31.

28. De Langhe S, Mulliez T, Veldeman L, et al. Factors modifying the risk for developing acute skin toxicity after whole-breast intensity modulated radiotherapy. BMC Cancer 2014;14:711.

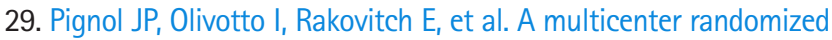
trial of breast intensity-modulated radiation therapy to reduce acute radiation dermatitis. J Clin Oncol 2008;26:2085-92.

30. Burnet NG, Johansen J, Turesson I, Nyman J, Peacock JH. Describing patients' normal tissue reactions: concerning the possibility of individualising radiotherapy dose prescriptions based on potential predictive assays of normal tissue radiosensitivity. Int J Cancer 1998;79:606-13.

31. Turesson I, Nyman J, Holmberg E, Oden A. Prognostic factors for acute and late skin reactions in radiotherapy patients. Int J Radiat Oncol Biol Phys 1996;36:1065-75.

32. Pires AM, Segreto RA, Segreto HR. RTOG criteria to evaluate acute skin reaction and its risk factors in patients with breast cancer submitted to radiotherapy. Rev Lat Am Enfermagem 2008;16:844-9.

33. Kraus-Tiefenbacher $U$, Sfintizky A, Welzel G, et al. Factors of influence on acute skin toxicity of breast cancer patients treated with standard three-dimensional conformal radiotherapy (3D-CRT) after breast conserving surgery (BCS). Radiat Oncol 2012;7:217.

34. Lilla C, Ambrosone CB, Kropp $\mathrm{S}$, et al. Predictive factors for late normal tissue complications following radiotherapy for breast cancer. Breast Cancer Res Treat 2007;106:143-50.

35. Barnett GC, De Meerleer G, Gulliford SL, Sydes MR, Elliott RM, Dearnaley DP. The impact of clinical factors on the development of late radiation toxicity: results from the Medical Research Council RT01 trial (ISRCTN47772397). Clin Oncol (R Coll Radiol) 2011;23:613-24.

36. Terrazzino $S$, La Mattina $P$, Masini $L$, et al. Common variants of eNOS and XRCC1 genes may predict acute skin toxicity in breast cancer patients receiving radiotherapy after breast conserving surgery. Radiother Oncol 2012;103:199-205.

37. Back M, Guerrieri M, Wratten C, Steigler A. Impact of radiation therapy on acute toxicity in breast conservation therapy for early breast cancer. Clin Oncol (R Coll Radiol) 2004;16:12-6.

38. Twardella D, Popanda O, Helmbold I, et al. Personal characteristics, therapy modalities and individual DNA repair capacity as predictive factors of acute skin toxicity in an unselected cohort 
of breast cancer patients receiving radiotherapy. Radiother Oncol 2003;69:145-53.

39. Porock D. Factors influencing the severity of radiation skin and oral mucosal reactions: development of a conceptual framework. Eur J Cancer Care (Engl) 2002;11:33-43.

40. Hymes SR, Strom EA, Fife C. Radiation dermatitis: clinical presentation, pathophysiology, and treatment 2006. J Am Acad Dermatol 2006;54:28-46.

41. Morganti AG, Digesu C, Panunzi S, et al. Radioprotective effect of moderate wine consumption in patients with breast carcinoma. Int J Radiat Oncol Biol Phys 2009;74:1501-5.

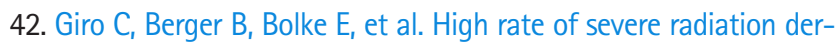

matitis during radiation therapy with concurrent cetuximab in head and neck cancer: results of a survey in EORTC institutes. Radiother Oncol 2009;90:166-71.

43. Bentzen SM, Harari PM, Bernier J. Exploitable mechanisms for combining drugs with radiation: concepts, achievements and future directions. Nat Clin Pract Oncol 2007;4:172-80.

44. Bowden SJ, Fernando IN, Burton A. Delaying radiotherapy for the delivery of adjuvant chemotherapy in the combined modality treatment of early breast cancer: is it disadvantageous and could combined treatment be the answer? Clin Oncol (R Coll Radiol) 2006;18:247-56. 